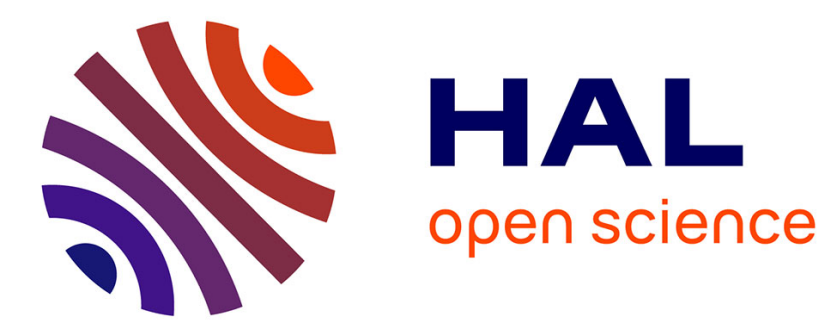

\title{
Pourquoi les Jeux de Sotchi seront plus coûteux que prévu
}

\author{
Wladimir Andreff
}

\section{To cite this version:}

Wladimir Andreff. Pourquoi les Jeux de Sotchi seront plus coûteux que prévu. La revue internationale et stratégique, 2013, 92 (hiver), pp.109-118. halshs-00971749

\section{HAL Id: halshs-00971749 \\ https://shs.hal.science/halshs-00971749}

Submitted on 3 Apr 2014

HAL is a multi-disciplinary open access archive for the deposit and dissemination of scientific research documents, whether they are published or not. The documents may come from teaching and research institutions in France or abroad, or from public or private research centers.
L'archive ouverte pluridisciplinaire HAL, est destinée au dépôt et à la diffusion de documents scientifiques de niveau recherche, publiés ou non, émanant des établissements d'enseignement et de recherche français ou étrangers, des laboratoires publics ou privés. 
Revue Internationale et Stratégique, ${ }^{\circ} 92$, hiver 2013, p. 109-118.

\title{
Pourquoi les Jeux de Sotchi seront plus coûteux que prévu
}

\author{
Wladimir Andreff \\ Professeur émérite à l'Université Paris 1 Panthéon-Sorbonne, président d'honneur de la \\ International Association of Sports Economists et de la European Sports Economics \\ Association.
}

Lorsque Sotchi l'emporte, en juillet $2007^{1}$, sur Pyeongchang et Salzbourg pour organiser les Jeux olympiques (JO) d'hiver de 2014, son dossier semble mirobolant et bien plus coûteux que les éditions précédentes. Entre l'obtention des Jeux et la cérémonie d'ouverture, la préparation de Sotchi est en proie, comme les Olympiades antérieures, à une hausse rapide des coûts, due à un phénomène économique de "malédiction du vainqueur » des enchères. Pour tous les JO d'été et d'hiver, les coûts réels au moment de la cérémonie d'ouverture ont dépassé les coûts annoncés lors de la candidature, à une exception près qui confirme la règle. On peut donc pronostiquer que les coûts seront dépassés à Sotchi en février 2014 ; d'ailleurs, ils le sont déjà.

\section{Les Jeux olympiques de Sotchi, un projet économique mirobolant ?}

Le projet des Jeux d'hiver de Sotchi annonçait, dans le dossier de candidature, un coût d'organisation de 1,2 milliard d'euros, pour lequel ont voté les membres du Comité international olympique (CIO). Mais il était indiqué que le Comité d'organisation local des JO (COJO) ne prendrait pas en charge le coût des investissements. Ceux-ci, hors budget du COJO, s'élevaient en 2007 à 8,4 milliards d'euros (12 milliards de dollars) pressentis, dont $60 \%$ à la charge de l'État russe. Ainsi, le coût total attendu des JO de Sotchi se situait d'emblée au-dessus de celui annoncé, organisation et investissements compris, dans les dossiers de candidature de Turin pour 2006 (4 milliards d'euros) et de Vancouver pour 2010 (2 milliards de dollars).

Les promoteurs du projet justifiaient un tel coût d'abord par l'investissement dans des équipements sportifs : 11 stades olympiques, stations de ski - dont la plus importante, Rosa Khutor - pour les épreuves de ski alpin, deux villages olympiques, une patinoire ouverte sur mer et montagne et des complexes balnéaires. Les adjectifs grandiloquents n'ont pas manqué. Ils s'appliquent encore plus aux infrastructures non sportives à mettre en place entre 2007 et février 2014. L'investissement pour l'aménagement du site de Sotchi est lourd : rénovation de quartiers entiers de la ville et de l'hôtel Rodina, construction d'un archipel (île de la Fédération) entièrement artificiel de 330 hectares, d'un nouvel aéroport doublant la capacité d'accueil, d'un nouveau port pour cargos, d'une deuxième voie ferrée de 67 kilomètres le long de la côte, de 580 kilomètres de route et d'une voie à grande vitesse entre l'aéroport et le site olympique (63 kilomètres), d'une autoroute de contournement de Sotchi, d'un métro aérien monorail de 50 kilomètres allant de la ville au site olympique, d'un centre de presse, de 5 complexes hôteliers de grand luxe, de deux centres commerciaux, d'une zone d'attractions touristiques, le creusement d'un nouveau tunnel routier, ainsi que des investissements pour atteindre une capacité hôtelière de 196000 chambres, augmenter la capacité de production

\footnotetext{
${ }^{1}$ Après l'échec de ses trois précédentes candidatures en 1989, 1993 et 2003. La première remontée mécanique a été construite à Sotchi en 1993.
} 
d'électricité de la zone et acheter des terrains à 3000 petits propriétaires expropriés, contre dédommagement, pour créer le parc olympique.

Pour actualiser le coût total de 9,6 milliards d'euros annoncé ex ante en 2007 et tenir compte de l'inflation ${ }^{2}$ que provoque toujours la préparation de l'accueil des Jeux, il est nécessaire de le majorer de $50 \%$, soit un coût total prévisible de 14,4 milliards d'euros courants à l'horizon 2014, contre un coût total effectif ex post de 7 milliards de dollars à Vancouver 2010. Un calcul économique correct devrait y ajouter un coût de maintenance des nouveaux équipements sur toute leur durée de vie. Malgré la crise financière qui a frappé la Russie en 2008, suivie d'une récession, le coût d'investissement des Jeux de Sotchi n'a cessé d'augmenter, selon diverses estimations, jusqu'à atteindre 33,3 milliards de dollars début 2010 et 51 milliards de dollars en février 2013.

Tableau $n^{\circ}$ 1: Coût ex ante et ex post des Jeux olympiques d'hiver

\begin{tabular}{|c|c|c|c|}
\hline $\begin{array}{l}\text { Ville, année } \\
\text { (Nombre de } \\
\text { candidats) }\end{array}$ & Coût ex ante (candidature) & Coût ex post (à l'ouverture) & Autre indice \\
\hline $\begin{array}{l}\text { Lake Placid } \\
1980 \\
\text { ( } 2 \text { candidats })\end{array}$ & $\begin{array}{l}\text { Coût d'opération COJO : } \\
47 \text { millions de dollars } \\
\text { Coût d'investissement : } \\
129 \text { millions de dollars }\end{array}$ & $\begin{array}{l}\text { Coût d'opération COJO : } \\
96 \text { millions de dollars }\end{array}$ & $\begin{array}{l}\text { Déficit olympique } \\
8,5 \text { millions de } \\
\text { dollars }\end{array}$ \\
\hline $\begin{array}{l}\text { Sarajevo } 1984 \\
(3 \text { candidats })\end{array}$ & $\begin{array}{l}\text { Coût d'opération COJO : } \\
\text { 17,6 millions de dollars }\end{array}$ & $\begin{array}{l}\text { Coût d'opération COJO : } \\
20,2 \text { millions de dollars } \\
\text { Coût d'investissement : } \\
\text { 15,1 millions de dollars }\end{array}$ & \\
\hline $\begin{array}{l}\text { Calgary } 1988 \\
(3 \text { candidats })\end{array}$ & $\begin{array}{l}\text { Coût total initial : } 500 \text { millions de } \\
\text { dollars canadiens }\end{array}$ & $\begin{array}{l}\text { Coût total : } 1 \text { milliard de dollars } \\
\text { canadiens } \\
\text { Coût d'opération COJO : } \\
636 \text { millions de dollars canadiens }\end{array}$ & \\
\hline Albertville 1992 & $\begin{array}{l}\text { Coût total initial : 2,933 milliards } \\
\text { de francs } \\
\\
1987: 3,160 \text { milliards de francs ; } \\
1991: 11,487 \text { milliards de francs } \\
\text { dont coût d'opération : } \\
3,233 \text { milliards de francs ; } \\
\text { équipements sportifs : } \\
714 \text { millions de francs } \\
\text { Infrastructures : } 8,630 \text { milliards de } \\
\text { francs } \\
\text { Coût d'hébergement : } \\
289 \text { millions de francs }\end{array}$ & $\begin{array}{l}\text { Coût d'opération COJO : } \\
4,200 \text { milliards de francs } \\
\text { équipements sportifs : } \\
5,755 \text { milliards de francs } \\
\text { infrastructures: } 7,8 \text { milliards de } \\
\text { francs } \\
\text { Coût d'hébergement: } 575 \text { millions } \\
\text { de francs }\end{array}$ & $\begin{array}{l}\text { Déficit olympique } \\
60 \text { millions de } \\
\text { dollars } \\
(285 \text { millions de } \\
\text { francs })\end{array}$ \\
\hline $\begin{array}{l}\text { Lillehammer } \\
1994\end{array}$ & $\begin{array}{l}\text { Coût total en } 1988: 1,511 \text { milliard } \\
\text { de dollars }\end{array}$ & Coût total : 1,7 milliard de dollars & $\begin{array}{l}\text { Déficit } \\
\text { olympique : } 343 \mathrm{~m}\end{array}$ \\
\hline
\end{tabular}

${ }^{2}$ Dans le dossier de candidature de Sotchi, le taux d'inflation est estimé à 5,91\% par an de 2007 à 2014, soit une augmentation de $50 \%$ du coût en euros courants en sept ans. Dans le secteur immobilier résidentiel, l'inflation a déjà dépassé $100 \%$ et les prix au mètre carré sont désormais supérieurs à ceux de Moscou. 


\begin{tabular}{|c|c|c|c|}
\hline (4 candidats) & & & llions de dollars \\
\hline $\begin{array}{l}\text { Nagano } 1998 \\
(5 \text { candidats })\end{array}$ & $\begin{array}{l}\text { Coût total en } 1992: 450 \text { millions } \\
\text { de dollars }\end{array}$ & $\begin{array}{l}\text { Coût total : } 875 \text { millions de } \\
\text { dollars }\end{array}$ & $\begin{array}{l}\text { Dette : } 11 \text { milliards } \\
\text { de dollars }\end{array}$ \\
\hline $\begin{array}{l}\text { Salt Lake City } \\
2002 \\
\text { (4 candidats) }\end{array}$ & $\begin{array}{l}\text { Coût d'opération : } 400 \text { millions de } \\
\text { dollars en } 1989 \text {; } \\
1996: 1 \text { milliard de dollars; } 1998 \\
\text { : 1,3 milliard de dollars }\end{array}$ & $\begin{array}{l}\text { Coût d'opération : 1,9 milliards de } \\
\text { dollars }\end{array}$ & $\begin{array}{l}\text { Déficit } \\
\text { olympique : } 168 \\
\text { millions de dollars }\end{array}$ \\
\hline $\begin{array}{l}\text { Turin } 2006 \\
\text { (6 candidats) }\end{array}$ & $\begin{array}{l}\text { Coût d'investissement: } \\
3,5 \text { milliards d'euros } \\
\text { Coût d'opération COJO : } 660 \\
\text { millions de dollars }\end{array}$ & $\begin{array}{l}\text { Coût d'investissement : } \\
13 \text { milliards d'euros } \\
\text { Coût d'opération COJO : } \\
1,357 \text { milliard de dollars }\end{array}$ & $\begin{array}{l}\text { Déficit olympique : } \\
38 \text { millions de } \\
\text { dollars }\end{array}$ \\
\hline $\begin{array}{l}\text { Vancouver } 2010 \\
\text { ( } 3 \text { candidats })\end{array}$ & $\begin{array}{l}\text { Coût d'opération COJO : } 846 \\
\text { millions de dollars ; } \\
\text { Révisé en } 2007: 1,63 \text { milliards de } \\
\text { dollars } \\
\text { Coût de construction : } \\
1,22 \text { milliard de dollars }\end{array}$ & $\begin{array}{l}\text { Coût d'opération COJO : } \\
\text { 1,88 milliard de dollars } \\
\text { Coût d'investissement: } \\
\text { 2,5 milliards de dollars } \\
\text { Coût total : } 3,569 \text { milliards de } \\
\text { dollars; en } 2010: 7 \text { milliards de } \\
\text { dollars }\end{array}$ & $\begin{array}{l}\text { Déficit olympique : } \\
37 \text { millions de } \\
\text { dollars }\end{array}$ \\
\hline $\begin{array}{l}\text { Sotchi } 2014 \\
\text { ( } 3 \text { candidats) }\end{array}$ & $\begin{array}{l}\text { Coût d'organisation : 1,2 milliard } \\
\text { d'euros } \\
\text { Coût d'investissement : } \\
12 \text { milliards de dollars } \\
2010: 33 \text { milliards de dollars ; } \\
2013: 51 \text { milliards de dollars }\end{array}$ & & \\
\hline
\end{tabular}

Source : Wladimir Andreff, « The Winner's Curse: Why Is The Cost of Mega Sporting Events so Often Underestimated ? », in Wolfgan Meanning et Andrew Zimbalist, International Handbook on the Economics of Mega Sporting Events, Cheltenham, Edward Elgar, 2012, mis à jour.

\section{La malédiction du vainqueur de l'enchère (winner's curse) ${ }^{3}$}

Le dépassement de coût est inéluctable avec le mode actuel d'attribution des JO. Le CIO a l'exclusivité de leur organisation et des symboles olympiques. Il a un monopole mondial absolu sur l'offre des Jeux et ouvre tous les quatre ans un appel à candidature qui s'apparente à un processus d'enchère dans lequel les villes candidates sont mises en concurrence. Le CIO en tire deux avantages : ses membres peuvent choisir - voter pour - le projet le plus intéressant, sûr, magnifique, tout en se déchargeant de l'essentiel des coûts sur la ville organisatrice, à part ceux pris en charge par le budget du COJO, et en n'assumant pas les investissements dans les infrastructures non sportives. Il est donc logique que les membres du CIO votent pour le projet le plus magnifique, ce qui se vérifie le plus souvent puisqu'ils choisissent en général le projet le plus coûteux ${ }^{4}$. Après le désastre financier de Montréal 1976 et le succès de Los Angeles 1984, le CIO a cherché à contrer ce biais en affichant que désormais, « les Jeux paieront les Jeux », formule qui garantit que le coût des JO sera financé, y compris in fine, par les finances locales, donc les contribuables. Mais ceci ne garantit en rien que les coûts ne soient pas dépassés.

${ }^{3}$ La même analyse plus approfondie est disponible dans Wladimir Andreff, «The Winner's Curse: Why Is The Cost of Mega Sporting Events so Often Underestimated ? », op. cit.

${ }^{4}$ Ibid. 
L'objectif de chaque ville candidate est évidemment d'obtenir l'organisation et, dans ce but, elle va surenchérir sur les projets des autres candidates, en proposant des cérémonies, des équipements olympiques et des infrastructures non sportives les plus attractives possibles. Or, ceci devrait alourdir le coût apparent affiché dans le dossier de candidature. Pour éviter de détériorer la qualité de son dossier par un coût prohibitif, chaque ville est incitée, pour remporter l'enchère, à «tricher »-au sens d'occulter en partie l'information - sur ses coûts, en sous-estimant sciemment les coûts et en surestimant les retombées positives attendues ${ }^{5}$. Le dossier de candidature de Londres 2012 minorait ainsi les dépenses de sécurité, négligeait le coût des paralympiques et oubliait d'imputer la TVA sur certaines dépenses. Pour faire valider cette sous-estimation du coût - et une surestimation des retombées économiques positives -, chaque ville candidate est infiniment désireuse qu'une étude préalable démontre un impact positif aussi important que possible de l'accueil des JO sur l'économie locale et avec le moindre coût possible. Elle est prête à payer le prix fort, de préférence à un prestigieux bureau d'études, pour obtenir une étude contenant cette conclusion favorable, quitte à ce qu'elle comporte des biais méthodologiques pour parvenir à ce résultat ${ }^{6}$. Ce que, sachant, le bureau fournira volontiers une étude minorant les coûts - et majorant l'impact positif-, condition pour obtenir à nouveau des contrats d'étude avec de prochaines villes candidates.

L'attribution de l'organisation des JO par enchère conduit donc les villes candidates à surenchérir les unes sur les autres, en acceptant finalement de payer trop cher pour offrir au $\mathrm{CIO}$ les plus beaux Jeux possibles. Ceci confirme un résultat connu de la théorie économique des enchères : chaque fois qu'une enchère porte sur un objet dont la valeur est incertaine ${ }^{7}$ mais la même pour tous les candidats, le vainqueur est celui qui a le plus surestimé la valeur de l'objet ${ }^{8}$. C'est le phénomène de la winner's curse ou malédiction du vainqueur de l'enchère. La ville candidate qui l'emporte accepte de payer des coûts trop élevés pour organiser les Jeux : comme elle les a sous-estimés dans son dossier de candidature, la réalité des coûts véritables va s'imposer à elle entre le jour de sa désignation comme ville hôte et le jour de l'ouverture des Jeux.

Deux précisions encore. Le budget du COJO étant financé par le CIO et la liste des équipements olympiques requis étant fixée à l'avance par celui-ci, la sous-estimation des coûts va surtout concerner les infrastructures non sportives, sur l'ampleur desquelles la ville cherche à faire la différence par rapport à ses concurrentes ; là va jouer l'essentiel de la winner's curse et du dépassement des coûts. D'autre part, l'attribution des JO est réalisée dans un contexte d'asymétrie d'information. Chaque ville candidate connaît son projet olympique dans les moindres détails, elle peut communiquer pour mettre en valeur ses aspects les plus avantageux tout en en laissant d'autres dans l'ombre, en particulier les coûts. Le CIO n'a pas

\footnotetext{
${ }^{5}$ Voir John L. Crompton, «Analysis of Sports Facilities and Events: Eleven Sources of Misapplication », Journal of Sport Management, vol. 9, $\mathrm{n}^{\circ}$ 1, 1995 ; Stéfan Késenne, « Do We Need an Economic Impact Study or a Cost-Benefit Analysis of a Sport Event? », European Sport Management Quarterly, vol. 5, n 2, 2005 ; Victor A. Matheson, «Economic Multiplier and Mega Event Analysis », International Journal of Sport Finance, vol. 4, $\mathrm{n}^{\circ}$ 1, 2009 ; et Philip K. Porter, « Mega-sports Events as municipal Investments: A Critique of Impact Analysis », in John L. Fiszel, Elisabeth Gustafson et Lawrence Hadley, Sports Economics: Current Research, Westport, Praeger, 1999.

${ }^{6}$ D'où la méfiance et les critiques méthodologiques des économistes envers les études d'impact réalisées pour les villes candidates, exhaustivement rassemblées dans Éric Barget et Jean-Jacques Gouguet, Événements sportifs. Impact économique et social, Bruxelles, De Boek, 2010. Voir aussi Wladimir Andreff, Mondialisation économique du sport. Manuel de référence en Économie du sport, Bruxelles, De Boeck, 2012.

${ }^{7}$ Personne ne connaît a priori la valeur marchande réelle du fait d'être désigné ville hôte des Jeux et surtout pas le (les) membre(s) du CIO.

${ }^{8}$ Voir Roger H. Thaler, The Winner's Curse: Paradoxes and Anomalies of Economic life, Princeton, Princeton University Press, 1994.
} 
les moyens de contrôler le détail des coûts pour chaque ville candidate, ni donc de déceler leur degré de sous-estimation. C'est seulement une fois qu'une ville est désignée comme hôte que le CIO envoie ses représentants visiter le site olympique ; ils pourront vérifier que le projet est en bonne voie, qu'il n'est pas en retard mais, à ce stade, il est trop tard pour empêcher que les coûts aient été préalablement sous-estimés ou même pour vérifier qu'il en a été ainsi. Une fois déclenché, le processus de la winner's curse ne peut être stoppé.

Si Sotchi a été désignée en 2007, c'est donc que la ville a sous-estimé ses coûts au moins autant, ou plus, que Pyeongchang et Salzbourg. Elle s'est embarquée, à coup sûr, dans un processus de dépassement inéluctable.

\section{Tous les Jeux olympiques ont coûté plus cher que prévu}

Après la désignation d'une ville organisatrice, la winner's curse se matérialise tout d'abord par une augmentation continue des devis, des dépenses et des coûts, disons au-dessus de $30 \%$ de majoration des coûts due à l'inflation $(50 \%$ dans le cas de Sotchi avec une inflation annuelle de $5,91 \%$ ). Le résultat est que le coût effectif ex post finit par être nettement supérieur (dépassement entre 30 et $50 \%$ ) au coût ex ante du dossier de candidature. Un second indice réside dans des révisions du projet olympique : la construction d'un édifice qui n'avait pas été envisagé dans le dossier de candidature, ce qui augmente les coûts, ou la suppression d'une infrastructure ou d'un équipement prévu, trop coûteux. Un troisième indicateur est un délai de réalisation supérieur à celui mentionné ex ante. C'est l'indice que le CIO surveille le plus attentivement, car tout doit être prêt le jour de la cérémonie d'ouverture. Or, il coûte toujours plus cher de réaliser dans l'urgence un investissement qui a pris du retard; les Jeux d'Athènes 2004 en sont la meilleure illustration récente. Deux indices complémentaires révèlent que la winner's curse est à l'œuvre : une rallonge de fonds publics ou une subvention additionnelle en provenance du budget municipal, régional ou de l'État ; l'augmentation du déficit budgétaire - ou la transformation d'un excédent budgétaire en déficit - et de l'endettement de la ville hôte pour financer le projet olympique.

En se cantonnant au premier et principal indice de la winner's curse, les coûts ont toujours été dépassés, le plus souvent de plus de $30 \%$, lorsque l'on observe tous les JO d'été depuis Munich 1972 et d'hiver depuis Lake Placid 1980, à une exception près. Sous réserve d'informations trop peu détaillées, Sarajevo 1984ㄹ, Lillehammer 1994 et Nagano 1998 ont moins sensiblement dépassé le coût annoncé que les autres villes hôtes des Jeux d'hiver. Le COJO a affiché un déficit (dépassement du coût d'organisation) à Lake Placid 1980, Albertville 1992, Lillehammer 1994 et Turin 2006. Albertville et Turin ont bénéficié de rallonges budgétaires. Avec un coût plus que doublé, Lake Placid 1980, Calgary 1988, Albertville 1992, Salt Lake City 2002, Turin 2006 et Vancouver 2010 - où le budget sécurité a été multiplié par 7 - vérifient la thèse de la winner's curse. Les données connues ${ }^{10}$ montrent que Sotchi 2014 est victime de la même malédiction, conclusion qui ne pourra être définitivement tirée qu'en février prochain.

Il existe une exception : les JO d'été de Los Angeles 1984, toujours cités en exemple par le CIO, se sont terminés par un bénéfice financier et, surtout, sans dépassement des coûts. Le coût d'organisation fut de 546 millions de dollars et le coût total, tous investissements compris, de 1592 millions de dollars ex post. Mais ex ante, Los Angeles 1984 n'avait pris aucun engagement vis-à-vis du CIO sur les coûts, il est donc logique qu'il n'y ait pas eu de dépassement. Ces Jeux avaient été attribués en 1977, juste après la révélation du désastre financier de Montréal 1976. Il n’y avait alors aucun candidat pour organiser l'édition 1984. Le

\footnotetext{
${ }^{9}$ Voir Boris Tihi, XIV Olimpijske Igre kao factor razvoja Sarajevskog regiona, Bosne I Hercegovine I sire zadjednice, Sarajevo, Ekonomski Institut Sarajevo, 1983.

${ }^{10}$ Voir tableau $\mathrm{n}^{\circ} 1$.
} 
CIO a donc dû convaincre Los Angeles d'être unique et seule candidate. N'ayant pas subi la surenchère de concurrents, Los Angeles n'a pas été victime de la winner's curse et du dépassement des coûts. C'est la meilleure exception possible pour confirmer la théorie énoncée. Elle est pleine d'enseignements pour la recherche de solutions au dépassement des coûts des JO.

\section{Sotchi 2014, et après ?}

Les JO de Sotchi vont inéluctablement dépasser les coûts annoncés et le mieux que puissent faire les responsables des dépenses - COJO et municipalité - est d'essayer de limiter l'ampleur du dépassement. Le record à battre dans la même olympiade - Londres 2012 - est plus qu'un quadruplement des coûts (11,6 milliards de livres de coûts ex post contre 2,4 milliards de livres annoncés au départ). En 2013, Sotchi reste dans la course en ayant déjà quadruplé le coût d'investissement. Pour Pyeongchang 2018 aussi, il est déjà trop tard. Mais après ? La question est complexe. On peut chercher à éradiquer les causes profondes de la winner's curse ou bien simplement à freiner le dépassement des coûts.

Sans modifier le mode d'attribution des Jeux, on peut suggérer que le CIO annonce qu'il sélectionnera le projet olympique le moins coûteux. Très improbable, même irréaliste car cela contredirait la stratégie du CIO d'obtenir les Jeux les plus magnifiques possibles. En outre, une telle formule renforcerait les causes de la winner's curse au lieu de les éradiquer. En effet, pour présenter le coût le plus bas, chaque ville serait incitée à tricher en sous-estimant davantage encore les coûts annoncés dans le dossier de candidature. À l'inverse, si le CIO affichait un plafond de coût maximum des $\mathrm{JO}$ à ne pas dépasser, pour freiner la tendance au gigantisme des Jeux, il recevrait des projets concurrents affichant tous ce coût maximum et il se priverait d'un critère économique de choix de la ville hôte.

On peut alors envisager que le $\mathrm{CIO}$ prenne à son compte une part plus grande du coût des JO, non seulement le budget du COJO et des équipements olympiques, mais aussi une partie des infrastructures non sportives. Ceci aurait un double effet : la richesse du CIO s'amoindrirait sachant que les revenus - des sponsors, des droits télévisés, etc. - tirés des JO forment la source essentielle de ses finances ${ }^{11}$, ce qui réduirait les avantages et le pouvoir des membres votants; ces derniers prendraient personnellement conscience du coût réel des JO, on pourrait alors espérer que dans leurs votes futurs, ils prennent moins en compte l'aspect mirobolant des projets et davantage les coûts véritables, en consultant les coûts ex post observés lors des Olympiades précédentes.

Un traitement plus radical du problème serait d'éradiquer les racines du dépassement des coûts en supprimant la mise aux enchères de l'organisation des Jeux entre plusieurs villes. Une solution absolue et définitive, sans doute utopique, serait de fixer une localisation unique des JO, une fois pour toutes, disons une pour les Jeux d'été, une pour les Jeux d'hiver. Cette solution fut un moment discutée pour le centenaire des JO, en 1996, en référence au site des tout premiers Jeux à Olympie, en Grèce. Les avantages seraient immenses : il n'y aurait plus d'enchères, plus de surenchères par sous-estimation des coûts et, en outre, il n'y aurait plus à construire à chaque olympiade de nouveaux équipements sportifs et de lourdes infrastructures ; il suffirait de rénover et moderniser l'existant, ce qui est beaucoup moins coûteux. Une telle solution a peu de chance de s'imposer car elle heurte trop d'intérêts économiques et financiers, qui ont beaucoup à gagner à chaque nouvelle localisation des $\mathrm{JO}$ : sponsors, chaînes de télévision, multinationales, bâtiment et travaux publics, transports, hôtellerie, restauration, etc., et avant tout le CIO lui-même.

\footnotetext{
${ }^{11}$ Jean-Loup Chappelet et Brenda Kübler-Mabbott, The International Olympic Committee and the Olympic System, Abingdon, Routledge, 2008.
} 
Une alternative serait de chercher une formule acceptable pour les intérêts susmentionnés et le CIO, qui éliminerait ou réduirait considérablement la winner's curse. Si l'on exclut la désignation unilatérale et discrétionnaire d'une ville hôte par le CIO - que celle-ci serait en droit de refuser-, on pourrait s'orienter vers une règle de rotation géographique, un peu comme celle, intercontinentale, pratiquée par la FIFA pour l'attribution de la Coupe du monde de football. En définissant des zones géographiques suffisamment étroites ${ }^{12}$ à chaque rotation, il n'y aurait guère que deux ou trois villes susceptibles, par leur taille urbaine et économique, d'accueillir les JO. La probabilité étant faible qu'elles soient toutes désireuses de candidater à une même date, le CIO se retrouverait souvent face à une seule ville candidate, condition de base pour la disparition de la winner's curse. Dans une hypothèse moins favorable, il resterait deux villes candidates et, alors, plutôt que de les départager par enchère, on suggère que le $\mathrm{CIO}$ trouve alors une procédure pour inciter - dont financièrement - l'une des deux villes à se désister et à soutenir sa voisine géographique.

\section{La prévision des médailles distribuées aux JO de Sotchi 2014}

Publié à la veille des JO de Pékin, un modèle économétrique ${ }^{13}$ testé sur les Jeux d'été depuis 1976 explique la répartition des médailles olympiques entre nations participantes à l'aide de cinq variables: le PIB par habitant de chaque nation, sa population, être le pays hôte des Jeux, avoir été naguère un pays à régime communiste, la culture sportive approximée par l'appartenance à une région particulière du monde. Utilisé pour la prévision, ce modèle a exactement «prédit » $70 \%$ des gains de médailles aux Jeux de Pékin, et même $88 \%$ des résultats si l'on accepte une marge d'erreur de deux médailles ${ }^{14}$. Adapté aux Jeux d'hiver et testé sur les données depuis 1964, le modèle explique les gains de médailles par pays par les quatre même premières variables que pour les Jeux d'été ; la cinquième variable - tous les pays ont une même culture de pratique des sports d'hiver dans ce cas - a été remplacée par deux autres : la durée annuelle d'enneigement du pays et le nombre de stations de sports d'hiver dans le pays. Utilisé en prévision, le modèle ainsi adapté annonce ${ }^{15}$ :

\begin{tabular}{|c|c|c|c|c|}
\hline Nations & Médailles & Médailles & Limite & Limite \\
\hline & en 2010 & prévues en 2014 & inférieure & supérieure \\
\hline États-Unis & 37 & 36 & 33 & 38 \\
\hline Allemagne & 30 & 28 & 26 & 30 \\
\hline Canada & 26 & 27 & 25 & 28 \\
\hline Russie & 15 & 24 & 21 & 27 \\
\hline Norvège & 23 & 24 & 22 & 25 \\
\hline
\end{tabular}

${ }^{12}$ Par exemple : Afrique subsaharienne, Afrique du Nord, Amérique du Nord, Amérique centrale, Amérique du Sud, Asie du Sud Est, Asie du Sud et centrale, Moyen-Orient, Europe du Nord, Europe de l'Ouest, Europe centrale et orientale, Océanie. Une ville ne pourrait espérer candidater qu'une fois par siècle mais sans enchère. Les ambitions de candidature se limiteraient aux plus raisonnables sur un horizon temporel très long.

${ }^{13}$ Madeleine Andreff, Wladimir Andreff et Sandrine Poupaux, «Les déterminants économiques de la performance sportive : Prévision des médailles gagnées aux Jeux de Pékin », Revue d’Économie Politique, vol. $118, \mathrm{n}^{\circ} 2,2008$.

${ }^{14}$ Wladimir Andreff, «Comparaison entre les prévisions et les médailles gagnées aux Jeux de Pékin », in Gilbert Avanzini, Thierry Maudet et Patrick Mignon (coord.), Pékin 2008 : Regards croisés sur la performance sportive olympique et paralympique, Paris, INSEP, 2009.

${ }^{15}$ Madeleine Andreff et Wladimir Andreff, «Economic Prediction of Medal Wins at the 2014 Winter Olympics », Ekonomika a Management, vol. 2011, n 2, février 2011. 


\begin{tabular}{|c|c|c||c|c|}
\hline Autriche & 16 & 15 & 14 & 16 \\
\hline Suède & 11 & 13 & 12 & 14 \\
\hline France & 11 & 12 & 11 & 13 \\
\hline Chine & 11 & 11 & 9 & 13 \\
\hline Corée du Sud & 14 & 11 & 10 & 13 \\
\hline
\end{tabular}

La Russie améliore fortement sa performance piteuse de Vancouver 2010, grâce à sa forte croissance économique de 1999 à 2007 et, surtout, à l'effet pays hôte. La prévision pour Sotchi 2014 est en dessous des espoirs des autorités russes : la première marche du podium. Si la Russie se situait à la limite supérieure de l'intervalle de confiance statistique, elle pourrait monter sur la troisième marche. Bien entendu, les prévisions du modèle ne doivent pas être prises pour certaines. Pour les JO de Pékin, l'énorme importance de l'effet pays hôte n'a pas été prévue et le modèle a sous-estimé les gains de médailles de la Chine. M. Poutine peut donc encore croiser les doigts. 